\title{
Female Peritoneal Tuberculosis with Ascites, Pelvic Mass, or Elevated CA 125 Mimicking Advanced Ovarian Cancer: A Retrospective Study of 26 Cases
}

\author{
Haijiao Wang1, Xinglong Qu², Xiaoxia Liu¹, Lili Ding ${ }^{3}$ and Ying Yue ${ }^{1}$
}

\begin{abstract}
This study aimed to summarise the clinical experience of peritoneal tuberculosis (TB). Clinical data of 26 patients admitted and diagnosed with peritoneal TB mimicking advanced ovarian cancer or peritoneal carcinoma between June 2008 and June 2017, was evaluated. The mean age was $35.92 \pm 15.30$ years. The most common findings were abdominal mass $(n=21,80.77 \%)$ and elevated CA 125 levels $(n=19,73.08 \%)$. One patient underwent PET-CT and was diagnosed with peritoneal TB. Laparotomy was performed in 14 patients (70\%) and laparoscopy in six patients (30\%), out of whom two patients were converted to laparotomy due to severe adhesions. Meanwhile, mass puncture biopsy or small incision biopsy was the preferred method for diagnosing peritoneal TB, while PET-CT was considered as a good diagnostic method.
\end{abstract}

Key Words: Peritoneal tuberculosis, Infection, Advanced ovarian cancer, Peritoneal carcinoma.

Tuberculosis (TB) is a chronic granulomatous infectious disease caused by Mycobacterium tuberculosis infection and commonly affects the respiratory system. Number of people infected with TB remains high, which led to about 2 million deaths, especially in developing countries. 1 Over the past two to three decades, various factors have caused the spread of TB worldwide, such as poor socioeconomic conditions, increase in incidence of HIV infection, and poor case findings. ${ }^{2}$ In 2016, the estimated global incidence was 10.4 million cases, and about 1.3 million TB-related deaths were reported. ${ }^{3}$ Thus, TB has a serious impact on public health.

Peritoneal TB is rare and affects about $1-2 \%$ of all cases. The diagnosis of peritoneal TB is easily mixed with advanced ovarian cancer (AOC) or peritoneal carcinoma because of overlapping laboratory and clinical findings, including elevated CA-125 levels, ascites and pelvic mass. ${ }^{4}$ There may be difficulty in diagnosing these possible diseases without pathological findings through noninvasive means. 5 Therefore, increasing the gynecologist's awareness regarding peritoneal TB and avoiding extended surgery is important.

Clinical data of 26 patients was retrospectively reviewed who were admitted and diagnosed with peritoneal TB mimicking advanced (AOC) or peritoneal carcinoma in this hospital between June 2008 and June 2017. Descriptive statistics was used. Mean \pm SD values for

Department of Gynecologic Oncologyl / Respiration ${ }^{2}$ /

Intensive Care Unit ${ }^{3}$, First Hospital of Jilin University,

Changchun, Jilin, China

Correspondence: Dr. Ying Yue, Department of Gynecologic

Oncology, First Hospital of Jilin University, Changchun, Jilin,

China

E-mail: yying119@126.com

Received: July 12, 2018; Accepted: November 23, 2018 age, CA 125 levels, length of hospital stay, and expenditure were calculated.

The symptoms of patients are shown in Table I. The mean age was $35.92 \pm 15.30$ years. For patients who underwent surgery, mean length of hospital stay was 11.7 \pm 5.03 days and the mean \pm SD expenditure was RMB $¥ 15618.86 \pm 7357.65$. For patients who did not undergo surgery, the mean length of hospital stay was $5 \pm 2$ days and the mean expenditure was RMB $¥ 7574.64 \pm 5089.66$.

Abdominal puncture was performed in 10 patients, 3 patients developed ascites after surgery, and 1 patient underwent pleural puncture to remove fluid from the pleural space. Cytological studies were negative for malignant cells in 12 patients; presence of lymphocytes, neutrophil cells, and mesothelial cells in 8 patients; and absence of epithelial cells in 1 patient. CA 125 levels were present in 21 patients $(21 / 26,80.77 \%)$ and elevated in 19 patients, with mean value of 382.92 $\pm 376.32 \mathrm{U} / \mathrm{ml}$. Two patients showed normal CA 125 levels, 6 patients showed CA 125 levels between 35 and $100 \mathrm{U} / \mathrm{ml}, 11$ patients showed CA 125 levels between 101 and $1,000 \mathrm{U} / \mathrm{ml}$, and 2 patients showed CA 125 levels higher than $1,000 \mathrm{U} / \mathrm{ml}$.

Table I: Clinical symptoms of peritoneal tuberculosis.

\begin{tabular}{lcc}
\hline Clinical symptoms & $\mathrm{n}$ & $\%$ \\
\hline Abdominal mass & 21 & 80.77 \\
Ascites & 15 & 57.69 \\
Abdominal distention & 15 & 57.69 \\
Abdominal pain & 11 & 42.31 \\
Fever & 9 & 34.62 \\
Infertility & 3 & 11.54 \\
Weight loss & 2 & 7.69 \\
Nausea & 2 & 7.69 \\
Fatigue and night sweats & 1 & 3.85 \\
Chest tightness and shortness of breath & 1 & 3.85 \\
Menstrual extension & 1 & 3.85 \\
Urinary irritation & 1 & 3.85
\end{tabular}


Abdominal or pelvic enhanced CT scan was performed in 15 patients and showed omentum and mesentery thickening and nodules $(12,80 \%)$, peritoneal thickening $(6,40 \%)$, ascites $(11,73.33 \%)$, masses in the unilateral $(3,20 \%)$ or bilateral adnexal area $(8,53.33 \%)$, pelvic and abdominal masses $(2,13.33 \%)$, lymph node enlargement $(5,33.33 \%)$, metastatic cancer $(5,33.33 \%)$, TB (2, $13.33 \%)$, and ovarian cancer $(1,6.67 \%)$. MRI was performed in 3 patients, and masses in the unilateral $(1,33.33 \%)$ or bilateral adnexa area $(1,33.33 \%)$, and omentum thickening and nodules $(1,33.33 \%)$ were observed. One patient underwent PET-CT and was diagnosed with peritoneal TB, pericardial effusion, pleural effusion, and ascites; abdominal CT scan was also performed, but the results were negative.

Twenty patients underwent surgery $(20 / 26,76.92 \%)$ and 6 patients did not undergo surgery $(6 / 26,23.08 \%)$. For patients who underwent surgery, laparotomy was performed in $14(14 / 20,70 \%)$ patients and laparoscopy in $6(6 / 20,30 \%)$ patients. However, two procedures were converted to laparotomy due to severe adhesion. Tuboovarian masses were observed in $8(8 / 20,40 \%)$ patients. Adhesions were found in $13(13 / 20,65 \%)$ patients. Multiple diffuse white miliary nodules were seen in $10(50 \%)$ patients. Ascites was observed in 6 $(6 / 20,30 \%)$ patients, while omentum contracture was seen in $1(1 / 20,5 \%)$ patient. Hydrosalpinx was seen in $2(2 / 10,20 \%)$ patients. Enlargement of the fallopian tubes was observed in $4(4 / 20,20 \%)$ patients. Tuboovarian abscess was seen in $2(2 / 20,10 \%)$ patients, and abdominal abscess was observed in $1(1 / 20,5 \%)$ patient because of coexistent teratoma rupture. Frozen section was performed in $16(16 / 20,80 \%)$ patients during the operation, and $14(14 / 16,87.5 \%)$ patients were diagnosed with TB, because a teratoma rupture with tuberculous failed to correct the diagnosis, and the other one.

Surgical procedures included total hysterectomy in $3(3 / 20,15 \%)$ patients, subtotal hysterectomy in $1(1 / 20$, $5 \%)$ patient, and salpingectomy in $10(10 / 20,50 \%)$ patients, including two left-sided and eight bilateral procedures. Biopsies were performed in 17 (17/20, 85\%) patients; peritoneum in $7(7 / 20,35 \%)$ patients, omentum in $3(3 / 20,15 \%)$, ovary in $5(5 / 20,25 \%)$, fallopian tube in $1(1 / 20,5 \%)$, and mass in $1(1 / 20,5 \%)$. Adnexectomy were performed in $3(3 / 20,15 \%)$ patients, of whom one had right adnexectomy and two had bilateral adnexectomy. Oophorocystectomy was performed in $4(4 / 20$, $20 \%$ ) patients: two had right oophorocystectomy and the other two had bilateral oophorocystectomy. All 26 patients received complete anti-tuberculous therapy in a specialised hospital and were cured.

For diagnosing peritoneal TB or AOC, imaging techniques like CT scan and MRI have been performed but have limited efficacy due to the diffuse nature of the disease and the presence of small implants. CT scan detected metastatic cancer in $33.33 \%(5 / 15)$ of the patients in our study. PET-CT as a non-invasive method has been used for diagnosing peritoneal TB; some studies proved its success in diagnosing peritoneal TB, whereas other studies reported its inability to diagnose peritoneal TB. Sharma et al. considered that PET-CT was not used in most of the cases reported in this study due to its high cost. ${ }^{6}$ In this study, only one patient underwent PET-CT scan and was diagnosed with peritoneal TB and avoided surgery. We also analysed the hospitalisation expenditures and found that the expenditure of patient who underwent PET-CT was RMB $¥ 6433.02$, which was close to the average expenditures of patients who underwent surgery. Thus, we presumed that PET-CT scan can help the clinicians to decide whether a surgery should be performed or not. Certainly, analysis of ascitic fluid obtained by paracentesis can also be used for definitive diagnosis. However, some gynecologists do not support it due to the risk of preoperative tumor rupture or poor prognosis in ovarian cancer patients.

Performing laparotomy may be justifiable to make a pathological diagnosis; some authors suggest that laparoscopy is a safe tool to obtain tissue biopsy and thus prevent extended surgery. ${ }^{7}$ However, in this study, two patients converted to laparotomy due to severe adhesion. Instead, frozen section is recommended and was performed in 16 patients in our study. However, it failed to diagnose two patients.

Only when noninvasive procedures do not lead to diagnosis, invasive methods should be considered for distinguishing peritoneal TB from AOC or peritoneal carcinoma. PET-CT may be a good diagnostic method, and the cost is not very high; however, further studies using PET-CT should be conducted to confirm this finding. Mass puncture biopsy or small incision biopsy is a method to be considered, especially for young adults who have fertility requirements and elderly patients who would be unable to tolerate radical surgery.

\section{REFERENCES}

1. Thaiss CA, Kaufmann SH. Toward novel vaccines against tuberculosis: Current hopes and obstacles. Yale J Biol Med 2010; 83:209-15.

2. Shim SW, Shin SH, Kwon WJ, Jeong YK, Lee JH. CT differentiation of female peritoneal tuberculosis and peritoneal carcinomatosis from normal-sized ovarian cancer. J Comput Assist Tomogr 2017; 41:32-8.

3. Fujiwara M, Kawasaki M, Hariguchi N, Liu Y, Matsumoto M. Mechanisms of resistance to delamanid, a drug for mycobacterium tuberculosis. Tuberculosis (Edinb) 2018; 108:186-94.

4. Drayer SM, Shank JJ. Infectious diseases mimicking ovarian carcinomatosis. Gynecol Oncol Rep 2018; 26:29-31.

5. Chhabra S, Saharan K, Pohane D. Pelvic tuberculosis continues to be a disease of dilemma-case series. Indian $J$ Tuberc 2010; 57:90-4.

6. Sharma JB, Jain SK, Pushparaj M, Roy KK, Malhotra N, Zutshi V, et al. Abdomino-peritoneal tuberculosis masquerading as ovarian cancer: A retrospective study of 26 cases. Arch Gynecol Obstet 2010; 282:643-8.

7. Bhargava DK, Chopra PS, Nijhawan S, Dasarathy S, Kushwaha AK. Peritoneal tuberculosis: laparoscopic patterns and its diagnostic accuracy. Am J Gastroenterol 1992; 87:109-12.

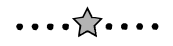

